
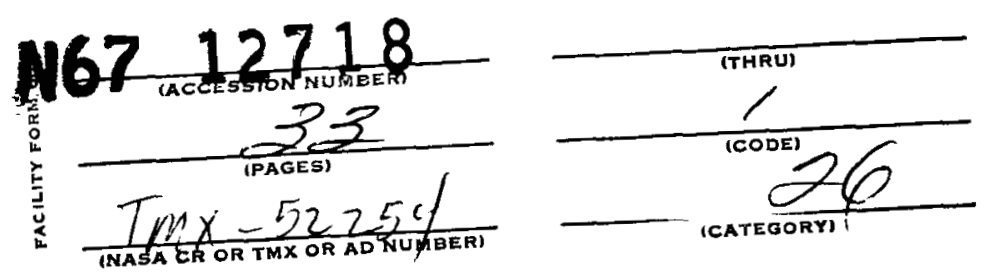

SPECTRAL EMISSIVITY OF HIGHLY DOPED SILICON

by Curt H. Liebert

Lewis Research Center

Cleveland, Ohio
GPO PRICE CFSTI PRICE(S)

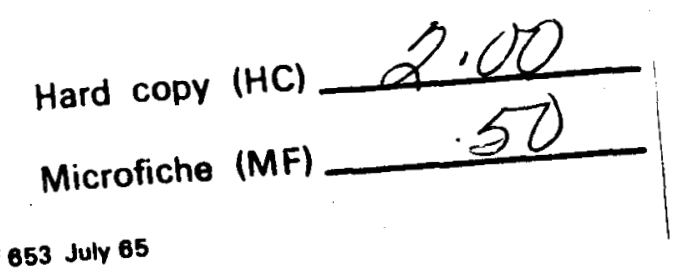

TECHNICAL PAPER proposed for presentation at Thermophysics

Specialists Conference sponsored by the American

- Institute of Aeronautics and Astronautics

New Orleans, Louisianna, April 17-19, 1967 


\title{
SPECTRAL EMISSIVITY OF HIGHLY DOPED SILICON
}

\author{
by Curt H. Liebert
}

Lewis Research Center

Cleveland, Ohio

TECHNICAL PAPER proposed for presentation at

Thermophysics Specialists Conference

sponsored by the American Institute of Aeronautics and Astronautics

New Orleans, Louisianna, April 17-19, 1967

NATIONAL AERONAUTICS AND SPACE ADMINISTRATION 


\author{
SPECTRAL EMISSIVITY OF HIGHLY DOPED SILICON \\ by Curt H. Liebert \\ Lewis Research Center \\ National Aeronautics and Space Administration \\ Cleveland, Ohio
}

\title{
SUMMARY
}

Measurements were made at temperatures of $300^{\circ}, 882^{\circ}$, and $1074^{\circ} \mathrm{K}$ of the spectral normal emissivity of opaque, highly doped silicon. The silicon was doped with arsenic and boron to $\mathrm{N}_{\mathrm{n}}=2.2 \times 10^{19}, 3.7 \times 10^{19}, 8.5 \times 10^{19}$ electrons $/ \mathrm{cm}^{3}$ and $\mathrm{N}_{\mathrm{p}}=6.2 \times 10^{19}, 1.4 \times 10^{20}$ holes $/ \mathrm{cm}^{3}$. The $300^{\circ} \mathrm{K}$ emissivity data were obtained at wavelengths from 2.6 to 35 microns. The higher temperature emissivities were measured from 3.5 to 14.8 microns.

Measurements of the carrier concentrations and direct current resistivity of the silicon were also performed. The carrier concentrations were determined from Hall measurements made at $300^{\circ} \mathrm{K}$. The direct current resistivity was measured at temperatures from $300^{\circ}$ to $1200^{\circ} \mathrm{K}$. These quantities (among others) were used in analytical calculations of the emissivities.

Agreement of the Hagen-Rubens theory with experiment was found at wavelengths greater than 12 microns and at $300^{\circ} \mathrm{K}$. Good agreement of the free carrier absorption theory with experiment was achieved at all wavelengths and temperatures investigated. 
The free carrier absorption theory predicts the emissivity in terms of the index of refraction and absorption index. The values of these quantities are presented. A comparison of the values of the absorption index obtained herein with those obtained from the literature showed good qualitative agreement.

\section{INTRODUCTION}

There is a considerable body of theory relating emissivity, absorbtivity, transmissivity, and reflectivity to the composition of homogeneous materials. Therefore, a better understanding of the optical properties of homogeneous materials could lead to a more complete insight of the properties required for radiant heat transfer calculations with a variety of materials. While a quantitative description of the latter may never be achieved, a fundamental understanding of the optical properties of a material may permit extrapolating measured data to temperatures or wavelengths beyond the convenient experimental range.

In recent years, there have been many theoretical at.tempts to explain the phenomenon of aispersion by relating the optical properties of smooth, homogeneous metals, dielectrics and semiconductors to electrical properties. Two approaches are the Hagen-Rubens theory (HR) (ref. 1) and the metallic or free carrier absorption theory (FCA) (ref. 2). 
The HR theory is based on a somewhat idealized physical model and leads to a simple expression for the normal spectral emissivity in terms of the wavelength and direct current resistivity. This theory is known to work well for metals at near room temperature and at wavelengths greater than about 10 microns. However, no investigations have been made in which this theory was applied to highly doped semiconductors.

The FCA theory is more general and the resulting equations for emissivity are necessarily more complex. This theory agrees well with the experimental optical properties of metals at wavelengths longer than 0.75 microns and over a: wide range of temperture. Good agreement has also been found between this theory and the optical properties of dielectrics when measured over a wide temperature range. Also, the FCA theory predicts well the room temperature emissivity of semiconductors. However, this theory has not been applied to the spectral emissivity of semiconductors at elevated temperatures.

In this investigation, the spectral normal emissivities of highly doped, single crystal, $n$ - and p-type silicon with optically smooth surfaces were experimentally determined in air at $300^{\circ}, 882^{\circ}$, and $1074^{\circ} \mathrm{K}$. Calculated emissivities as predicted by the HR and FCA theories were then compared to the experimental results. 
The variation of direct current resistivity with temperature and the room temperature Hall coefficient were also experimentally determined. The resistivity was used in both theories to calculate the emissivity. The Hall coefficient was used to determine the carrier concentration. The carrier concentration was used in the FCA theory along with other parameters chosen from the literature.

The results presented herein show that the HR theory agrees with experiment only at long wavelengths and room temperature whereas the FCA theory agrees at all wavelengths and temperatures investigated.

\section{SYMBOLS}

e electron or hole charge, $1.6 \times 10^{-19}$ coul

$k_{\lambda} \quad$ spectral absorption index

$\mathrm{m}$ mass of electron or hole, $9.11 \times 10^{-31} \mathrm{~kg}$

$\mathrm{m}^{*} \quad$ effective mass of electron or hole, $\mathrm{kg}$

$\mathrm{m}_{\mathrm{n}}^{*}$ effective mass of electron, $\mathrm{kg}$

$\mathrm{m}_{\mathrm{p}}^{*} \quad$ effective mass of hole, $\mathrm{kg}$

$\mathrm{N}$ carrier concentration, $\mathrm{cm}^{-3}$

$\mathrm{N}_{\mathrm{n}}$ electron carrier concentration, electrons $/ \mathrm{cm}^{3}$

$\mathrm{N}_{\mathrm{p}}$ hole carrier concentration, holes $/ \mathrm{cm}^{3}$

$\mathrm{n}_{\lambda}$ spectral index of refraction

$\mathrm{R}_{\mathrm{H}} \quad \mathrm{Hall}$ coefficient, $\mathrm{cm}^{3} / \mathrm{coul}$

$r_{\lambda}$ spectral normal reflectivity 
$\alpha_{\lambda} \quad$ spectral absorption coefficient, $\mathrm{cm}^{-1}$

$\epsilon_{\lambda} \quad$ spectral normal emissivity

$\epsilon_{\lambda, \exp }$ experimental spectral normal emissivity

$\epsilon_{\infty} \quad$ relative dielectric constant in the absence of any contribution from free charges at very high frequencies

$\epsilon_{\lambda, \max }$ maximum values of spectral normal emissivity

$\epsilon_{0} \quad$ permittivity of free space, $8.85 \times 10^{-12} \mathrm{coul}^{2} / \mathrm{nt} \mathrm{m}^{2}$

$\lambda \quad$ wavelength, $\mu$, microns

$\mu \quad$ microns

$\left.\begin{array}{ll}\mu_{\mathrm{p}} & \text { mobility of holes, } \\ \mu_{\mathrm{n}} & \text { mobility of electrons, }\end{array}\right\} \mathrm{sec}$ coul $/ \mathrm{kg}, \mathrm{m}^{2} / \mathrm{volt} \mathrm{sec}$

$v \quad$ frequency, $\sec ^{-1}$

$\rho \quad$ direct current resistivity, ohm-cm, sec

$\sigma \quad$ conductivity, $(\mathrm{ohm}-\mathrm{cm})^{-1},(\mathrm{sec})^{-1}$

$\tau \quad$ relaxation time, sec

$\omega \quad$ frequency, radians $/ \mathrm{sec}$

\section{APPARATUS AND PROCEDURE}

\section{Silicon Emissivity}

Two types of spectrophotometers were used. A PerkinElmer model 13 recording infrared spectrophotometer with an emittance-reflectance attachment was used to measure the normal spectral emissivity of specimens in air at temperatures of $882^{\circ}$ and $1074^{\circ} \mathrm{K}$. These measurements were taken 
over a wavelength range from 3.5 to 14.8 microns. The instrument was operated in the double beam mode with data recorded continuously on a strip chart recorder. The procedure for obtaining this data and the accuracy of the measurements are discussed in appendix A.

A Perkin-Elmer Model 521 spectrophotometer with a specular reflectance attachment was used to measure the near normal specular reflectivity in air at $300^{\circ} \mathrm{K}$ and wavelengths of 2.5 to 35 microns. (The angle of incidence of the light beam was about $6^{\circ}$ ). This reflectivity was determined by comparing the energy reflected by the samples to that which was reflected by an aluminum mirror. It was assumed that the mirror had a reflectivity of 100 percent. The normal spectral emissivity was calculated from the measured normal spectral reflectivity of the sample and the relation, $\epsilon_{\lambda}=1-r_{\lambda}$

Sample Preparation for Emissivity Measurements

Ingots of single crystal silicon doped with arsenic to achieve electron carrier concentrations of $2.2 \times 10^{19}, 3.7 \times 10^{19}$ and $8.5 \times 10^{19}$ electrons $/ \mathrm{cm}^{3}$ and doped with boron to achieve hole carrier concentrations $6.2 \times 10^{19}$ and $1.4 \times 10^{20}$ holes $/ \mathrm{cm}^{3}$ were purchased from the Allegheny Electron Chemicals Company. Disks 23 millimeters in diameter and 1.6 millimeters thick 
were cut from the ingots. The front side of the samples was optically polished and etched. Observations of the polished surface with an electron microscope showed that the width of ridges produced from the polishing procedures was about one-half micron. Two chromel-alumel thermocouples fabricated from 0.13 millimeter wire were mounted in a small groove sandhlasted into the back side of each sample. The thermocouple junctions were spot welded to the silicon. The thermocouple wires were held in the groove with a high temperature cement.

Transmittance measurements were performed on samples which were optically polished on both surfaces. The measurements established that the samples were opaque at wavelengths of 2.5 to 35 microns and at thicknesses of 23 millimeters.

\section{RESULTS AND DISCUSSION}

Room Temperature Resistivity and Carrier Concentration The values of carrier concentration $N=1 / R_{H^{e}}$, and resistivity were obtained for the silicon samples using conventional Hall and direct current resistivity apparatus and procedures. The values of resistivity obtained herein agreed within 5 percent of the data supplied by the manufacturer. An error analysis of the Hall and resistivity apparatus indicated that the measurements of carrier concentration and resistivity are accurate to 1 percent. 
Variation of Direct Current Resistivity with Temperature

The general variation of the resistivity of doped silicon with temperature is shown in the insert of figure 1. As the temperature rises, the resistivity decreases at low temperatures (region I), increases at moderate temperatures (region II) and again decreases at the higher temperatures (region III).

This investigation was performed in region II where the silicon resistivity increases as the temperature rises. Figure 1 presents data showing the variation of resistivity with temperature and carrier concentration for both the $n-$ and p-type semiconductors. The rising resistivity with increasing temperature is attributed to a decreasing mobility of charge carriers (ref. 3). This concept is visualized from equations

$$
\rho=\left(N_{n} e \mu_{n}\right)^{-1}=\left(N_{p} e \mu_{p}\right)^{-1}
$$

where the subscripts $\mathrm{n}$ and $\mathrm{p}$ refer to electrons and holes, respectively, and $\mu$ is the mobility. The carrier concentration in these heavily doped semiconductors is a constant with temperature over the range of $300^{\circ}$ to $1100^{\circ} \mathrm{K}$ (ref. 3). Therefore, as $p$ increases, $\mu_{n}$ or $\mu_{p}$ decreases. Reference 3 presents extensive data on the variation of resistivity with temperature for highly doped $n$ - and $p$-type silicon. When possible, a comparison was made between the experimental resistivity obtained herein and the data of reference 3 . The comparison showed agreement within \pm 3 percent. 
Comparison of the Hagen-Rubens Theory with Experiment Figures 2 and 3 present the experimental spectral normal emissivity of the three n-type and two p-type silicon specimens at temperatures of $300^{\circ}, 882^{\circ}$, and $1074^{\circ} \mathrm{K}$. As mentioned previously, the carrier concentration does not vary within this range of temperature, and thus is a constant for each figure. Included in these figures is the variation of spectral normal emissivity with wavelength and temperature as interpreted by the Hagen-Rubens theory which is based on the direct current resistivity. The equation for emissivity derived from the HR theory and Kirchoff's law is given for opaque solids as (ref. 1):

$$
\epsilon_{\lambda}=1-\left[\frac{2\left(\frac{\sigma}{\nu}\right)+1-2 \sqrt{\frac{\sigma}{\nu}}}{2\left(\frac{\sigma}{\nu}\right)+1+2 \sqrt{\frac{\sigma}{v}}}\right]
$$

where $\sigma=1 / p$ and $v$ is the frequency of the light corresponding to the wavelength, $\lambda$. The resistivity data shown in figure 1 was used to calculate the Hagen-Rubens emissivity from equation (1) at the appropriate wavelength, temperature, and electron concentration. Figures 2 and 3 show that the best agreement of experiment and theory was achieved at the highest values of $\mathrm{N}_{\mathrm{n}}$ or $\mathrm{N}_{\mathrm{p}}$ in the wavelength region of 12 to 35 microns and at room temperature. As $N_{n}$ or $\mathrm{N}_{\mathrm{p}}$ decreases, the agreement at room temperature is good only at progressively greater wavelengths. 
The calculated emissivity does not agree with the data taken from 3.5 to 14.8 microns and at the elevated temperatures. However, the curves may asymptotically approach the calculated values at the longer wavelengths (see fig. 2(a)).

\section{Comparison of the Free Carrier Absorption}

Theory with Experiment

Free carifer absorption thenry. - The inherent assumption which causes the HR theory to breakdown at short wavelengths for metals, and presumably for semiconductors, is that the period of the light is large as compared with the relaxation time (ref. 1). A more general theory which does not make this assumption, and which therefore can predict the experimental emissivity of silicon over a wider range of wavelengths is known as the Free Carrier Absorption Theory (FCA) (ref. 2). This theory predicts the emissivity in terms of the index of refraction, $n_{\lambda}$, and the absorption index, $k_{2}$.

For an opaque polished solid the spectral normal emissivity is given from Kirchoff's law and Fresnel's formulas as

$$
\epsilon_{\lambda}=\frac{4 n_{\lambda}}{\left(n_{\lambda}+1\right)^{2}+k_{\lambda}^{2}}
$$

Values of $n_{\lambda}$ and $k_{\lambda}$, can be related through the FCA theory to known or measurable quantities by the following equations: 


$$
\left.\begin{array}{l}
n_{\lambda}^{2}-k_{\lambda}^{2}=\epsilon_{\infty}-\frac{N e^{2}}{m^{*} \epsilon_{0}}\left(\frac{\tau^{2}}{1+\omega^{2} \tau^{2}}\right) \\
n_{\lambda} k_{\lambda}=\frac{N e^{2}}{2 \omega m^{*} \epsilon_{0}}\left(\frac{\tau}{1+\omega^{2} \tau^{2}}\right)
\end{array}\right)
$$

where $e$ is the electron or hole charge, $m^{*}$ is the effective mass of electrons or holes, $N$ is the carrier concentration of electrons or holes, $\epsilon_{O}$ is the permittivity of free space, $\epsilon_{\infty}$ is the relative dielectric constant in the absence of any contribution from free charges at very high frequencies, $\lambda$ is the wavelength, and $\tau$ is the relaxation time.

Sources of values of parameters. - The physical constants $e$ and $\epsilon_{0}$ are very accurately known. The carrier concentration $\mathrm{N}$ was determined from the experimental Hall measurements mentioned on page 7 ..

The free electron effective mass $m_{n}^{*}$, was taken from reference 4 as $0.27,0.26$ and $0.28 \mathrm{~m}$ for $\mathrm{N}_{\mathrm{n}}=2.2 \times 10^{19}$, $3.7 \times 10^{19}$ and $8.5 \times 10^{19}$ electrons $/ \mathrm{cm}^{3}$, respectively. These values were obtained from reflectivity measurements (presumably obtained at room temperature) which were then used to calculate $m_{n}^{*}$ from equations similar to (3) and an $\epsilon_{\infty}=11.7$. It is also shown in reference 4 that an $\mathrm{m}_{\mathrm{n}}^{*}=0.26 \mathrm{~m}$ may be calculated from cyclotron resonance experiments. Also, an $\mathrm{m}_{\mathrm{n}}^{*}=0.29 \mathrm{~m}$ may be obtained from the 
diamagnetic susceptibility of the free carriers (ref. 4). Thus, varying types of experiments produce values of $m_{n}^{*}$ which agree well. However, values of $\mathrm{m}_{\mathrm{n}}^{*}=0.43 \mathrm{~m}$ to $0.44 \mathrm{~m}$ for $\mathrm{N}_{\mathrm{n}}=6.4 \times 10^{19}$ to $1.1 \times 10^{20}$ electrons $/ \mathrm{cm}^{3}$ have also been published (ref. 4).

A value of $m_{p}^{*}=0.40 \mathrm{~m}$ was used in this investigation for the hole effective mass. Spitzer and Fan (ref. 5) determined $\mathrm{m}_{\mathrm{p}}^{*}=0.37 \mathrm{~m}$ from reflectivity measurements using a specimen with a hole concentration of $9.6 \times 10^{18}$ holes $/ \mathrm{cm}^{3}$. Reference 2 also shows that cyclotron resonance experiments produced a $\mathrm{m}_{\mathrm{p}}^{*}=0.39 \mathrm{~m}$ for holes.

Very little is known about the variation of effective mass with temperature. Thus, $\mathrm{m}_{\mathrm{n}}^{*}$ and $\mathrm{m}_{\mathrm{p}}^{*}$ were not varied with temperature in these experiments.

It is very difficult to experimentally determine $\epsilon_{\infty}$. Thus, data for the parameter are very scarce. Reference 6 shows that values have been measured for silicon that vary from 11.7 to 14.6. A value of $\epsilon_{\infty}=13 \pm 12$ percent spans this variation. A value of $\epsilon_{\infty}=13$ was used herein. Reference 7 indicates that the variation of $\epsilon_{\infty}$ with temperature may be small. Cardona, Paul and Brooks found a 2 percent increase in $\epsilon_{\infty}$ from about $70^{\circ}$ to $300^{\circ} \mathrm{K}$. Thus, no attempt was made to vary $\epsilon_{\infty}$ with temperature. 
The relaxation time was calculated from the direct current resistivity data given in figure 1 , the values of $\mathrm{m}^{*}, \mathrm{~N}$ and $\mathrm{e}$ discussed above and the following equation:

$$
\tau=\frac{\mathrm{m}^{*}}{\rho N \mathrm{e}^{2}}
$$

Comparison of FCA theory with experiment. - All the values of the parameters given on the right side of equation (3) have been established and thus the theoretical emissivity may be calculated. Figures 4 and 5 present the variation of spectral normal emissivity with wavelength and temperature as interpreted by the FCA theory. Also included in figures 4 and 5 are the same experimental emissivity data presented in figures 2 and 3 .

The variation of the theoretical emissivity from that of the experimental data obtained from the $n$-and $p$-type silicon at room temperature varies from about \pm 16 percent at $\epsilon_{\lambda \text {,exp }}=0.19$ to \pm 5 percent at $\epsilon_{\lambda \text {,exp }}=0.80$. This is the magnitude of the emissivity experimental error (see appendix A).

A divergence of experiment with the FCA theory was noted at the elevated temperatures. In general, the best agreement was obtained on the short wavelength side of the emissivity maximum, $\epsilon_{\lambda, \max }$. On the long wavelength side of $\epsilon_{\lambda, \max }$ the theory predicts emissivity values somewhat 
smaller than experiment; the theoretical emissivity is at most lower than the experimental results by about 14 percent. These discrepancies are probably due to the small variations of $\epsilon_{\infty}$ and $\mathrm{m}^{*}$ with temperature which were not considered. Values of ${ }_{7}$ and $k_{\lambda}$. Figures 6 and 7 present the values of $n_{\lambda}$ and $k_{\lambda}$ which were calculated with the FCA theory.

Spitzer and Fan (ref. 8) give values of the absorption coefficient, $\alpha_{\lambda}=4 \pi k_{\lambda} / \lambda$, calculated from measured values of room temperature reflectivity and transmissivity of $n$-and p-type silicon doped to carrier concentrations ranging from about $10^{16}$ to $10^{19} \mathrm{~cm}^{-3}$. Their data, which was obtained only on the short wavelength side of $\epsilon_{\lambda \text {, max }}$, show that plots of $\log \alpha_{\lambda}$ against $\log \lambda$ and plots of $\log \alpha_{\lambda}$ against $\log$ $\mathrm{N}$ is a straight line at wavelengths greater than 5 microns. Figure 8 presents the variation of $\log \alpha_{\lambda}$ against $\log$ $\lambda$ as calculated with the FCA theory at $\mathrm{N}_{n}=2.2 \times 10^{19}$ electrons $/ \mathrm{cm}^{3}$ and as calculated with the theory of reference 8 at $\mathrm{N}_{\mathrm{n}}=1 \times 10^{19}$ electrons $/ \mathrm{cm}^{3}$. Figure 8 shows that the values of $\log \alpha_{\lambda}$ obtained from both theories vary linearly with $\log \lambda$.

Limited calculations using the values of $k_{\lambda}$ and $N$ obtained herein at 6 microns also showed a linear variation of $\log \alpha_{\lambda}$ against $\log N$. 


\section{Interpretation of Results}

The primary result of these experiments is that the spectral normal emissivity of heavily doped silicon heated to temperatures up to $1075^{\circ} \mathrm{K}$ agrees well with the FCA theory. Therefore, use of the FCA theory will also predict the variations of $\epsilon_{\lambda}$ with wavelength, carrier concentration and temperature shown in figures 4 and 5 . The manner in which the use of the FCA theory predicts these experimental variations will now be discussed.

Effects of wavelength on $\epsilon_{\lambda}$. - The experimental emissivity of all silicon specimens passes through a maximum as the wavelength is varied. Calculated values of $\epsilon_{\lambda}$ using the FCA theory vary in a similar manner. This result can be interpreted as a coupling of $n_{\lambda}$ and $k_{\lambda}$ through equation (2).

Effect of carrier concentration on $\epsilon_{\lambda}$. - The emissivity increases as $N$ decreases and as $t$ increases at a a constant temperature and at given wavelength $(\lambda \geqq 12 \mu)$. An example of this effect at 14 microns and $1074^{\circ} \mathrm{K}$ is shown in table I.

Effect of temperature on $\epsilon_{\lambda}$ at long wavelengths. - The emissivity increases as the temperature increases and as $\tau$ decreases at a constant $\mathrm{N}$ and at wavelengths much longer than those wavelengths corresponding to $\epsilon_{\lambda \text {, max }}$. An example of this effect at 14 microns and at $N_{n}=8.5 \times 10^{19}$ electron $/ \mathrm{cm}^{3}$ is shown in table II. 
Effect of temperature on $\epsilon_{\lambda}$ at short wavelengths. Little variation of $\epsilon_{\lambda}$ with temperature occurs at constant $N$ and on the short wavelength side of $\epsilon_{\lambda \text {, max }}$. The low values of $k_{\lambda}$ calculated at wavelengths less than those for $\epsilon_{\lambda, \max }$ (fig. 6) contribute little to $\epsilon_{\lambda}$. Since $n_{\lambda}$ appears both in the numerator and denominator of equation (2), changes in its value with temperature cause relatively little change in the values ot $\epsilon_{\lambda}$.

\section{CONCLUDING REMARKS}

Measurements were taken at $300^{\circ}, 882^{\circ}$, and $1074^{\circ} \mathrm{K}$ of the spectral normal emissivity of heavily doped silicon. The silicon was doped with arsenic and boron to $N_{n}=2.2 \times 10^{19}, 3.7 \times 10^{19}, 8.5 \times 10^{19}$ electrons $/ \mathrm{cm}^{3}$ and $\mathrm{N}_{\mathrm{p}}=6.2 \times 10^{19}, 1.4 \times 10^{20}$ holes $/ \mathrm{cm}^{3}$. The $300^{\circ} \mathrm{K}$ emissivities were determined from 2.5 to 35 microns and the higher temperature emissitivies were measured from 3.5 to 14.8 microns. The carrier concentration at $300^{\circ} \mathrm{K}$ was determined from HaII measurements. Also, the resistivity of the silicon was measured at temperatures from $300^{\circ}$ to $1200^{\circ} \mathrm{K}$. The carrier concentrations and resistivities were used in analytical calculations of the emissivities.

The emissivity data were compared with the Hagen-Rubens equation which is based on the direct-current resistivity. This equation is known to predict well the emissivity of 
metals at long wavelengths and moderate temperatures. Measured resistivities were used to calculate this theoretical emissivity. Agreement of theory and experiment was found at wavelengths longer than 12 microns and at room temperature. The experimental emissivities were also compared with the calculated values using the free carrier absorption theory $(F C A)$. This theory considers $n_{\lambda}$ and $k_{\lambda}$ to be functions of $e, m^{*}, N, \epsilon_{0}, \epsilon_{\infty}, \lambda$, and $\tau$. The constants $e$ and $\epsilon_{0}$ are accurately known. The relaxation time is a function of $\rho, \mathrm{N}, \mathrm{m}^{*}$ and $e$. The experimental values of $\rho$ and $N$ determined herein were used to determine $\tau$. The values of $\mathrm{m}^{*}$ were obtained from the literature. The value of $\epsilon_{\infty}$, as determined by other experiments, is not known as accurately as other parameters. Thus, it was necessary to choose $\epsilon_{\infty}$ within the range of values given in the literature such that the theoretical variation of emissivity with wavelength could be more closely fitted to the experimental variations of $\epsilon_{\lambda}$.

Good agreement of the FCA theory and experiment was achieved for both the p-type and n-type silicon at all wavelengths and temperatures investigated. Therefore, it was concluded that the FCA theory as applied to silicon with $N$ greater than $10^{19} \mathrm{~cm}^{-3}$ is a proper physical law for predicting and interpreting the experimental variations of $\epsilon_{\lambda}$ with wavelength, carrier concentration and temperature observed in these experiments. 
It was generally found that the emissivity calculated with the FCA theory: (1) passed through a maximum as the wavelength varied; (2) varied little with temperature at a constant value of $\mathrm{N}$ and at all wavelengths corresponding to the short wavelength side of $\epsilon_{\lambda}$, max; (3) increased as $\mathrm{N}$ decreased and as $\tau$ increased at a constant temperature and at wavelengths of about 12 microns or greater; (4) increased as the temperaturc increased and as $\tau$ decreased at a constant $\mathrm{N}$ and at wavelengths much longer than those wavelengths corresponding to $\epsilon_{\lambda}$, max .

The index of refraction $\left(n_{\lambda}\right)$ and absorption index $\left(k_{\lambda}\right)$ of the silicon specimens at temperatures of $300^{\circ}, 882^{\circ}$, and $1074^{\circ} \mathrm{K}$ were also obtained using the FCA theory. The values of $k_{\lambda}$ obtained from the FCA theory at $300^{\circ} \mathrm{K}$ were used to calculate $\alpha_{\lambda}$. These values of $\alpha_{\lambda}$ were compared with those in the literature which are based on the optical ray absorption theory (ref. 8). Good qualitative agreement was obtained from this comparison. Thus, it was concluded that the FCA theory can be used with reasonable accuracy to obtain the $n_{\lambda}$ and $k_{\lambda}$ of opaque media. Also, it is expected that high temperature optical properties of highly doped germanium cadmium sulfide and alloys of silicon and germanium may also be obtained from the free carrier absorption theory. 


\section{APPENDIX A}

The uncertainty associated with the type of emissivity measurements made herein has been discussed by many authors. A good discussion covering most of the possible errors is given in reference 9 . One such error arises from a mismatch between the specimen temperature and the hohlraum temperature.: The difference can arise because the thermocouples are not recording the true specimen temperature and because of intolerable temperature gradients in the hohlraum. The following discussion will show how the errors were minimized in these experiments.

\section{Emittance of Standard}

Alumina, flame sprayed to a thickness of 0.4 millimeter on a roughened stainless steel 304 substrate was used as a standard to achieve a temperature match for this system. Thermocouples were added to the substrate in the manner previously discussed for silicon. The results of experiments by others measuring normal spectral emittance or reflectance agree that $\mathrm{Al}_{2} \mathrm{O}_{3}$ either in powdered, flame-sprayed, or sintered form has an emittance of 0.95 to 0.99 at 8.5 microns over a range of temperatures from about $300^{\circ}$ to $1300^{\circ} \mathrm{K}$ and over a wide range of surface roughness and thickness. A spectral emittance of the standard of 0.97 to 0.99 at 8.5 microns was achieved with the hohlraum and standard temperature settings 
given in table III. (The hohlraum has an advertized emittance of 0.99$)$. The lower indicated standard temperatures as compared to the hohlraum temperatures are attributed to heat conduction through the thermocouples and a measured temperature gradient through the standard of $2^{0}$ at about $880^{\circ}$ and $4^{\circ}$ at about $1070^{\circ} \mathrm{K}$. It is seen that the hohlraum used in these experiments possesed a temperature gradient of $3^{\circ}$ at about $890^{\circ}$ and $15^{\circ}$ at about $1090^{\circ} \mathrm{K}$. These small temperature gradients were achieved by inserting a ceramic plug into the hole in the hohlraum normally occupied by the reflectance sample holder.

Figure 8 shows the spectral normal emittance of the standard from about 1.2 to 14.8 microns at the temperature settings given in table III and also at $300^{\circ} \mathrm{K}$. The reflectivity attachment on the Perkin-Elmer Model 13 spectrophotometer was used to obtain the room temperature emittance. The good agreement of the data from about 1 to 2.5 microns and from about 4 to 14.8 microns indicates that a proper temperature match between hohlraum and standard has been established.

The room temperature data shown in figure 9 was found to agree well with room temperature measurements obtained by J. C. Richmond on flame-sprayed alumina (ref. 10). This agreement included complete verification of the emittance peak shown at about 3 microns (fig. 9). This peak is generally considered to be caused by absorbed water. Thus, the 
variations of $\epsilon_{\lambda}$ with temperature at wavelengths of about 2.5 to 4 microns are probably caused by more absorbed water present in the alumina at $300^{\circ}$ than at $1074^{\circ} \mathrm{K}$.

High Temperature Emissivity of Silicon

The temperature settings given in table III as obtained with the alumina standard were used to obtain the spectral normal emissivity of the various silicon samples. However, good emissivity data may be obtained from the silicon specimens by using the standard as reference only if the surface temperatures are about equivalent and the heat conduction loss along the thermocouple wires are about the same for both materials.

The temperature gradient across a silicon specimen with $\mathrm{N}_{\mathrm{n}}=2.2 \times 10^{19}$ electrons $/ \mathrm{cm}^{3}$ was experimentally found to be $3^{\circ}$ at about $880^{\circ}$ and $5^{\circ}$ at about $1070^{\circ} \mathrm{K}$. Thus, the temperature drop across the silicon and the standard are within $I^{\circ} \mathrm{K}$.

The heat loss through the thermocouple wires could not be determined experimentally and therefore a rough estimate of this loss was made analytically. Theory (ref. 11) shows that the thermocouple wires attached to the standard and the silicon may indicate different (and low) temperatures even though the specimens may, in fact, be at identical temperatures. The reason for this is that the heat conducted along 
the thermocouple wires welded to the stainless steel (standard) can be different from that conducted along the wires welded to the silicon. However, it was calculated that there was an identical heat loss along the thermocouple wires attached to either the silicon or the stainless steel. It was also calculated from the theory of reference 11 that the thermocouples would read about $20^{\circ} \mathrm{K}$ lower than the true specimen temperature at temperatures of $900^{\circ}$ to $1100^{\circ} \mathrm{K}$. This error is very close to that shown in table III. It is well known that the silicon surface will oxidize when exposed to air and that this oxidation is more rapid at higher temperatures. The oxidation, if sufficiently rapid, could cause large inaccuracies in the emissivity data. To evaluate the effect of oxidation on emissivity, measurements were made of the room temperature emissivity before and after the emissivity was measured in air at the elevated temperatures. Negligible variations of the room temperature emissivity were observed.

The above discussion has indicated that the experimental error associated with the high temperature silicon emissivity is small. This experimental error is considered to be between \pm 4 and \pm 7 percent.

The hohlraum and standard temperatures given in table III were averaged to obtain the average temperature given in table III. These temperatures are $882^{\circ}$ and $1074^{\circ} \mathrm{K}$. All 
the silicon properties needed to establish the correspondence between theory and experiment were evaluated at these two average temperatures and at room temperature $\left(300^{\circ} \mathrm{K}\right)$.

\section{Room Temperature Reflectivity}

The room temperature, specular, near normal reflectivity of all the silicon specimens was measured at wavelengths of 2.5 to 35 microns. For comparison purposes, the total (specular plus diffuse) reflectivity of the silicon from 3.5 to 14.8 microns was also measured using the Gier-Dunkel type hohlraum on the Perkin-Elmer Model 13 spectrophotometer. It was found that the specular and total reflectivity measurements generally, varied from \pm 16 percent at a: specular $\epsilon_{\lambda}=0.19$ to: \pm 5 percent at a specular. $\epsilon_{\lambda} \doteq 0.80$. Therefore, the room temperature specular reflectivity is believed to be be accurate at least to these percentages.

Howarth and Gilbert, reference 4, experimentally determined the room temperature spectral reflectivity of highly doped n-type silicon for purposes of determining the free electron effective mass. A comparison of their data obtained at wavelengths of 3.5 to 14.8 microns with the data obtained herein indicated good agreement.

\section{REFERENCES}

1. Born, Max and Wolf, Emil, Principles of Optics (The Macmillan Company, New York, 1964). 
2. Moss, T. S., Optical Properties of Semi-Conductors (Butterworths Scientific Publications, London, 1961).

3. Chapman, P. W., Tufte, O. N., Zook, J. D., and Long, D.,

"Electrical Properties of Heavily Doped Silicon,"

J. Appl. Phys. 34, 3291-3295 (1963) .

4. Howarth, I. E. and Gilbert, J. F., "Determination of

Free Electron Effective Mass on n-type Silicon,"

J. App. Phys. 34, 236-237 (1963) .

5. Spitzer, W. G. and Fan, H. Y., "Determination of Optical

Constants and Carrier Effective Mass of Semiconductors,"

Phys. Rev. 106, 882 (1957).

6. Runyan, W. R., Silicon Semiconductor Technology (McGraw Hill Book Co, Inc., New York, 1965).

7. Cardona, M., Paul, W., and Brooks, H., "Dielectric Constant Measurements in Germanium and Silicon at Radio Frequencies as a Function of Temperature and Pressure," Solid State Physics in Electronics and Telecommunications, M. Desirant and J. L. Michiels, editors (Academic Press, New York, 1960), Vol. 1, part 1, p. 206.

8. Spitzer, W. and Fan, H. Y., "Infrared Absorption in ntype Silicon," Phys. Rev. 108, 268-271 (1957).

9. Streed, E. R., McKeller, L. A., Polling, R., Jr., and Smith C. A., Errors Associated with Hohlraum Radiation Characteristics Determinations. Measurement of Thermal Radiation Properties of Solids. NASA SP-31, 1963, pp. 237-252. 
10. Liebert, C. H., "Spectral Emittance of Aluminum Oxide and Zinc Oxide on Opaque Substrates. NASA TN D-3115 (Novermber 1965).

11. Chapman, A. J., Heat Transfer (The Macmillian Company, New York, 1960). 
TABLE I. - VALUES OF $\epsilon_{\lambda}$ AND

$\tau$ CALCULATED AT 14 MICRONS

\begin{tabular}{|c|c|c|}
\hline \multicolumn{3}{|c|}{ AND $1074^{\circ} \mathrm{K}$} \\
$\begin{array}{c}\mathrm{N}_{\mathrm{n}} \times 10^{19}, \\
\text { electrons } / \mathrm{cm}^{3}\end{array}$ & $\epsilon \lambda$ & $\begin{array}{c}\tau \times 10^{-14} \\
\mathrm{sec}\end{array}$ \\
\hline 8.5 & 0.46 & 0.39 \\
3.7 & .62 & .47 \\
2.2 & .69 & .55 \\
\hline $\mathrm{N}_{\mathrm{p}} \times 10^{19}, \mathrm{~m}^{19}$ & $\epsilon \lambda$ & $\begin{array}{c}\tau \times 10^{-14} \\
\mathrm{sec}\end{array}$ \\
\hline 14 & 0.49 & 0.29 \\
6.2 & .62 & .39 \\
\hline
\end{tabular}

TABLE II. - VALUES OF $\epsilon_{\lambda}$ AND $\tau$ CALCULATED AT

14 MICRONS AND

$\mathrm{N}_{\mathrm{n}}=8.5 \times 10^{19}$

electrons $/ \mathrm{cm}^{3}$

\begin{tabular}{|c|c|c|}
\hline $\begin{array}{c}\text { Temperature, } \\
\text { OK }\end{array}$ & $\epsilon_{\lambda}$ & $\begin{array}{c}\tau \times 10^{-14} \\
\mathrm{sec}\end{array}$ \\
\hline 300 & 0.24 & 1.06 \\
1074 & .46 & 0.39 \\
\hline
\end{tabular}


TABLE III. - STANDARD AND HOHLRAUM TEMPERATURE SETTINGS NECESSARY TO ACHIEVE $\epsilon_{\lambda}=0.97 \pm 0.03$ AT 8.5 MICRONS

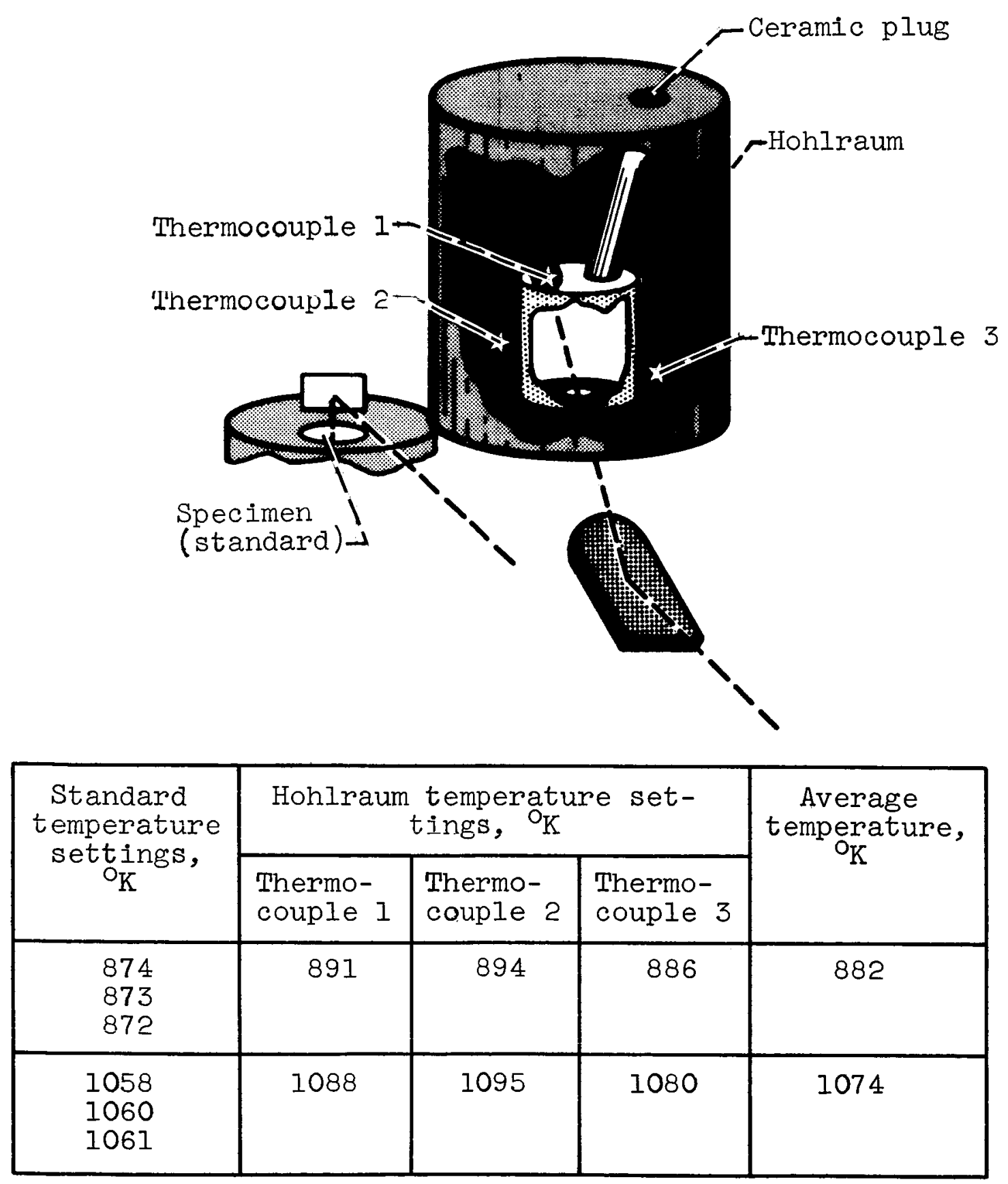




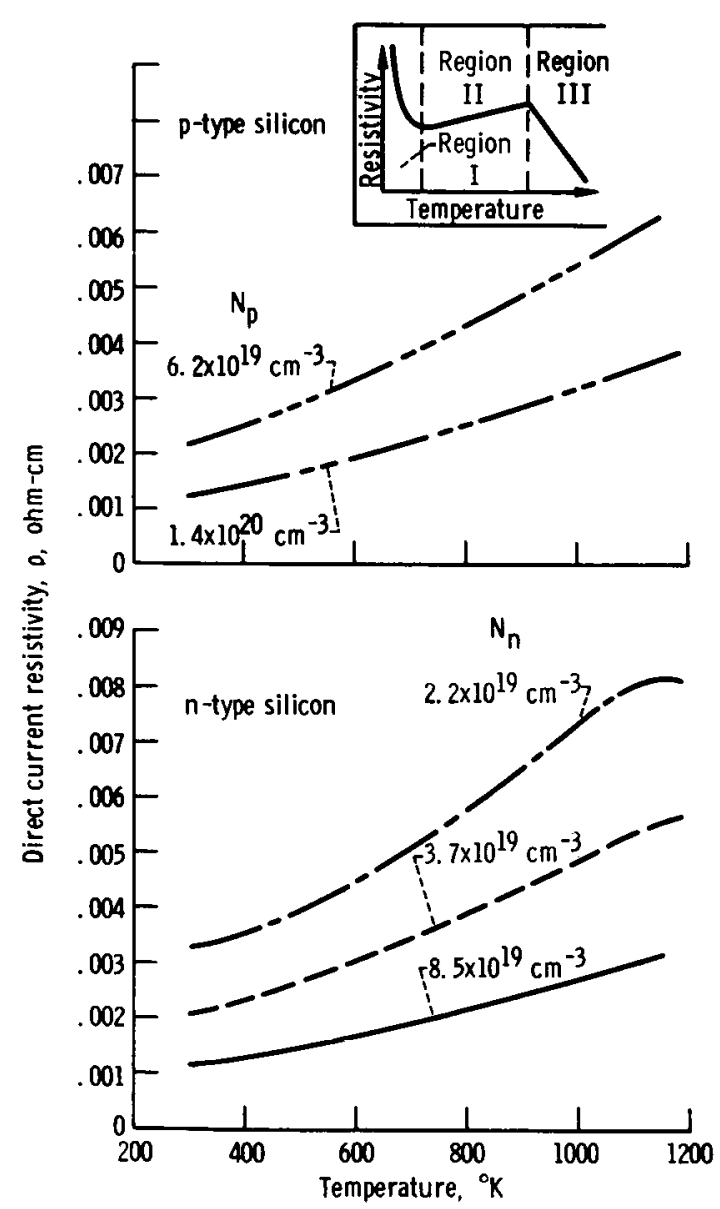

Figure 1. - Direct current resistivity of $n$ - and p-type silicon.

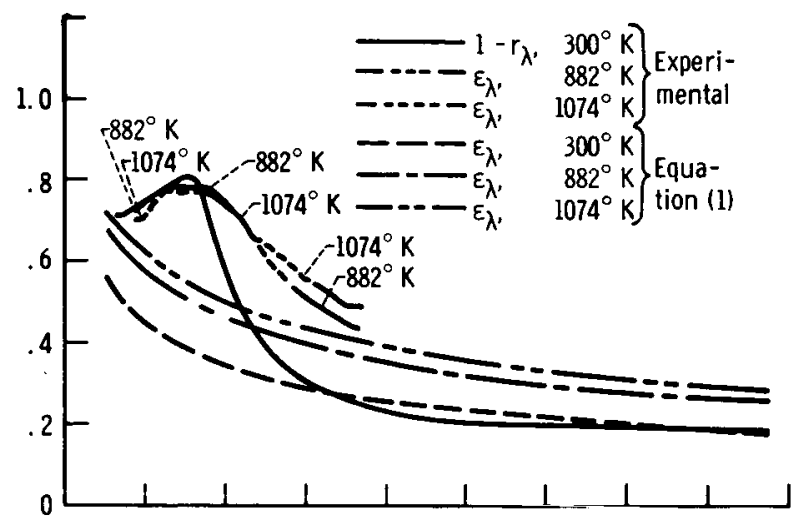

Figure 2(a). - Comparison of HR theory with experiment, $N_{n}=8.5 \times 10^{19}$ electrons $/ \mathrm{cm}^{3}$.

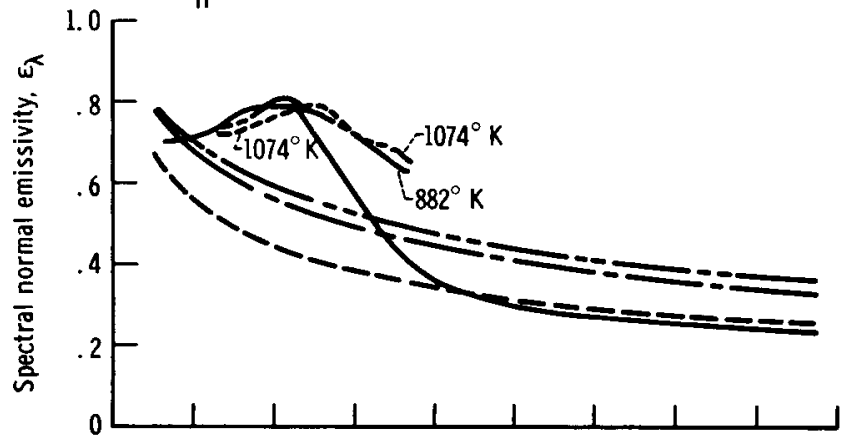

Figure 2(b). - Comparison of $H R$ theory with experiment, $N_{n}=3.7 \times 10^{19}$ electrons $/ \mathrm{cm}^{3}$.

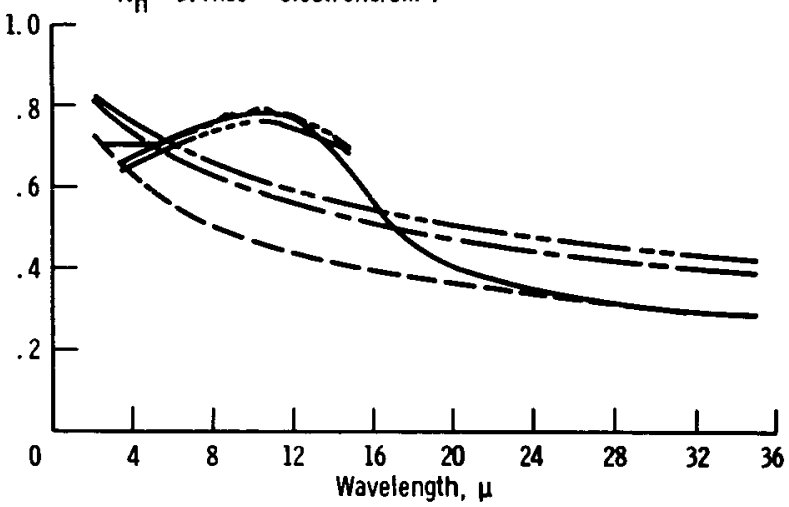

Figure $2 \mathrm{Cc}$. - Comparison of HR theory with experiment, $\mathrm{N}_{\mathrm{n}}=2.2 \times 10^{19}$ electrons $/ \mathrm{cm}^{3}$. 
T્木

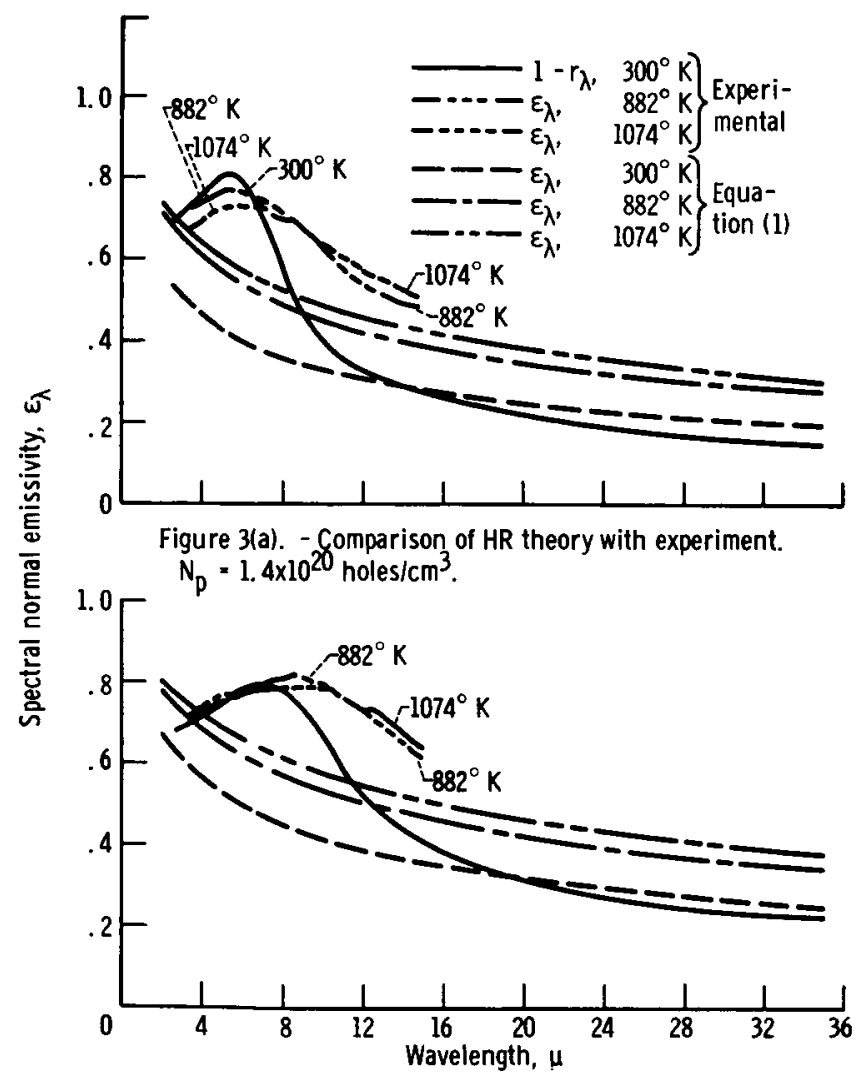

Figure 3(b). - Comparison of HR theory with experiment. $N_{p}=6.2 \times 10^{19}$ holes $/ \mathrm{cm}^{3}$.
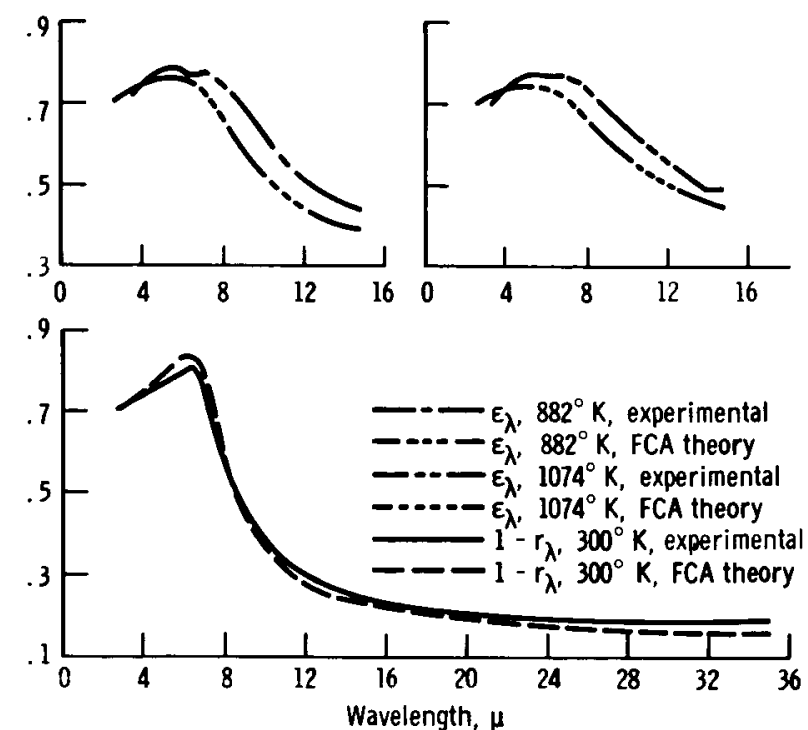

Figure 4(a). - Comparison of FCA theory with experiment.

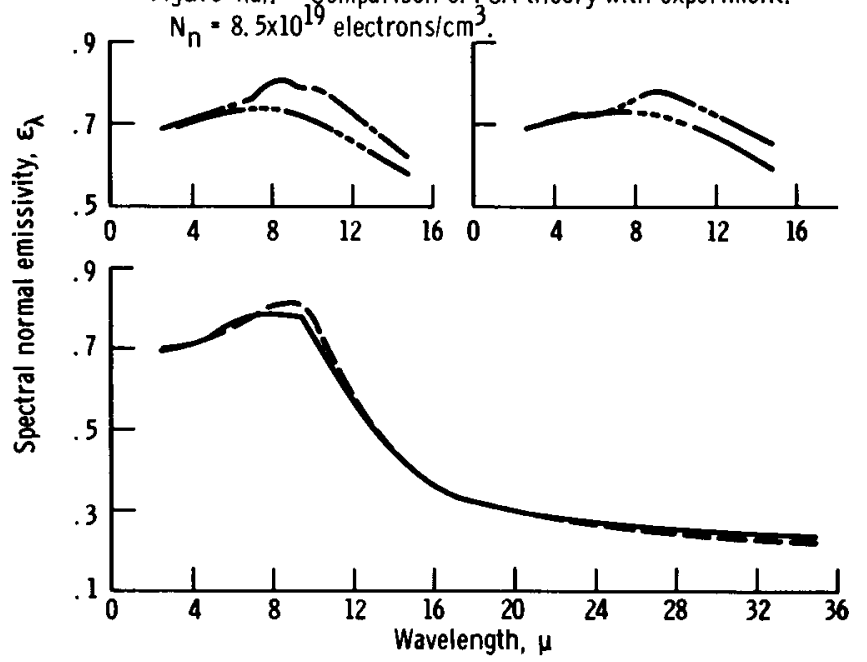

Figure 4(b). - Comparison of ECCA theory with experiment. $N_{n}=3.7 \times 10^{19}$ electrons $/ \mathrm{cm}^{3}$.
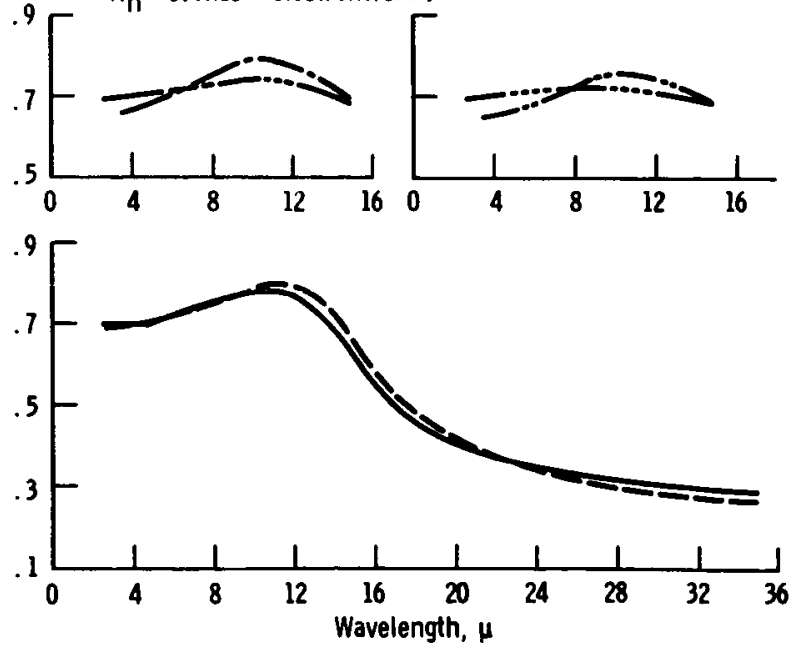

Figure $4(C)$. - Comparison of $F C A$ theory with experiment. $N_{n}=2.2 \times 10^{19}$ electrons $/ \mathrm{cm}^{3}$. 


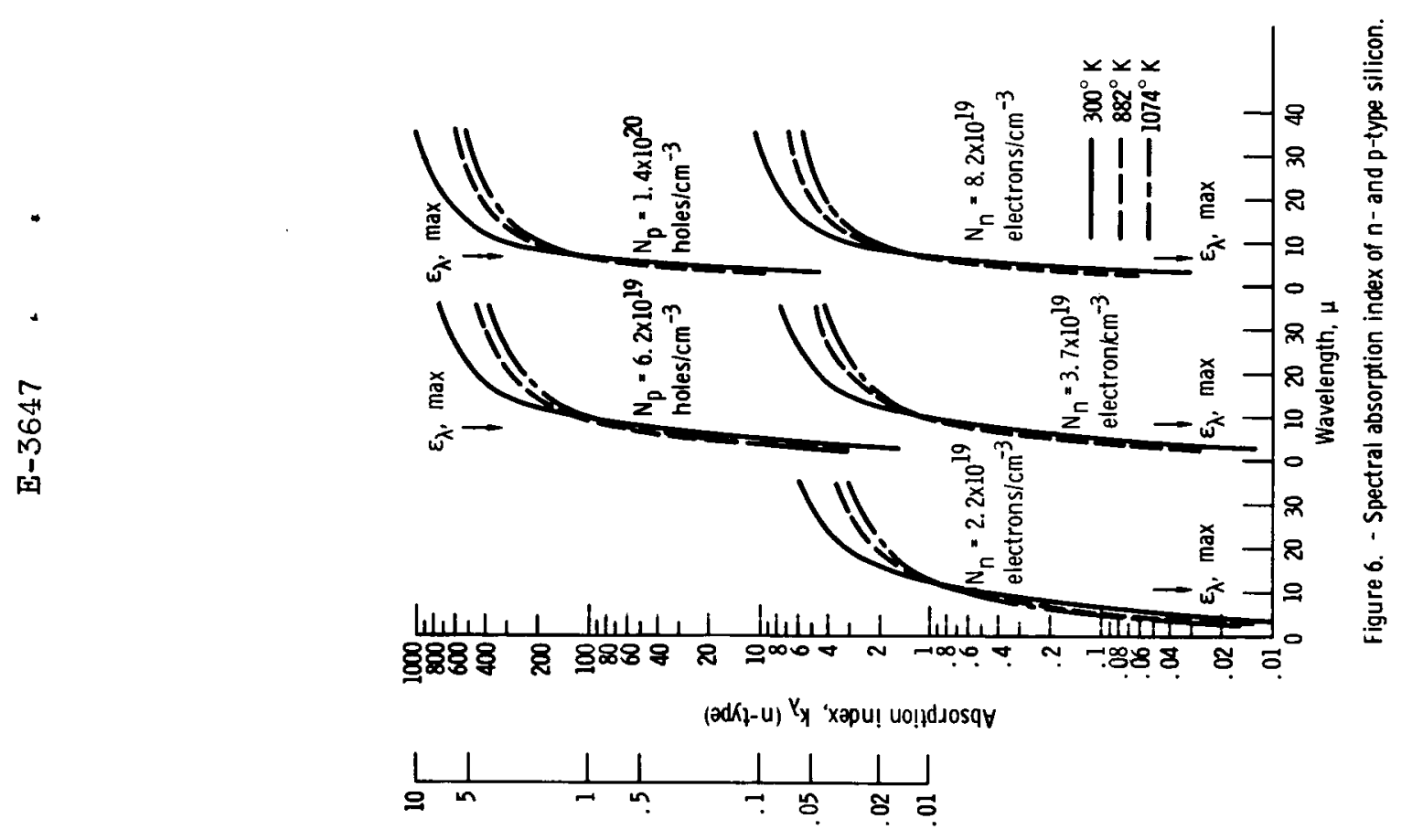

$\left(\operatorname{ad} X_{t}-d\right) Y_{y}$ 'xzpu! uo!jdsosqy

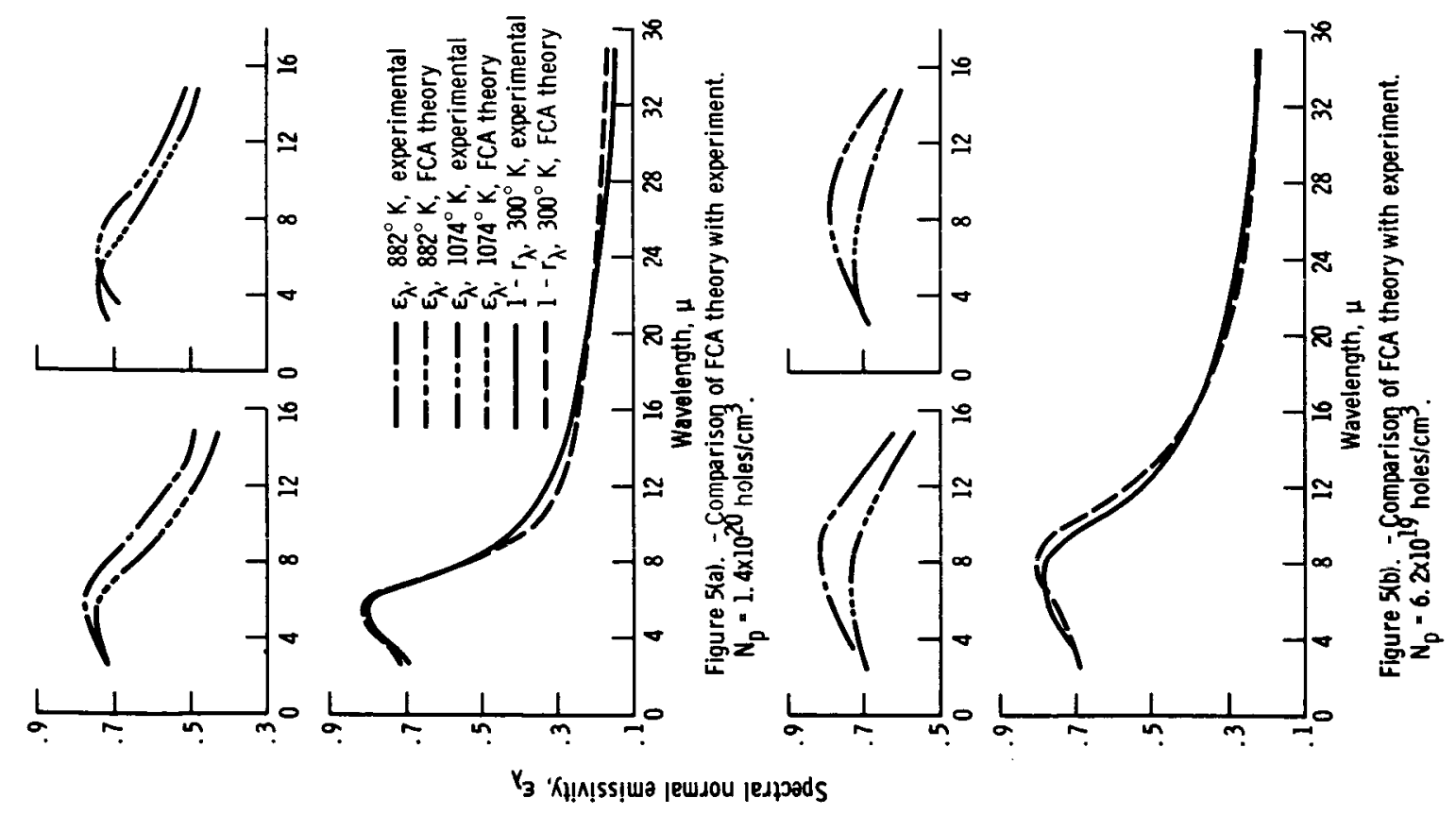



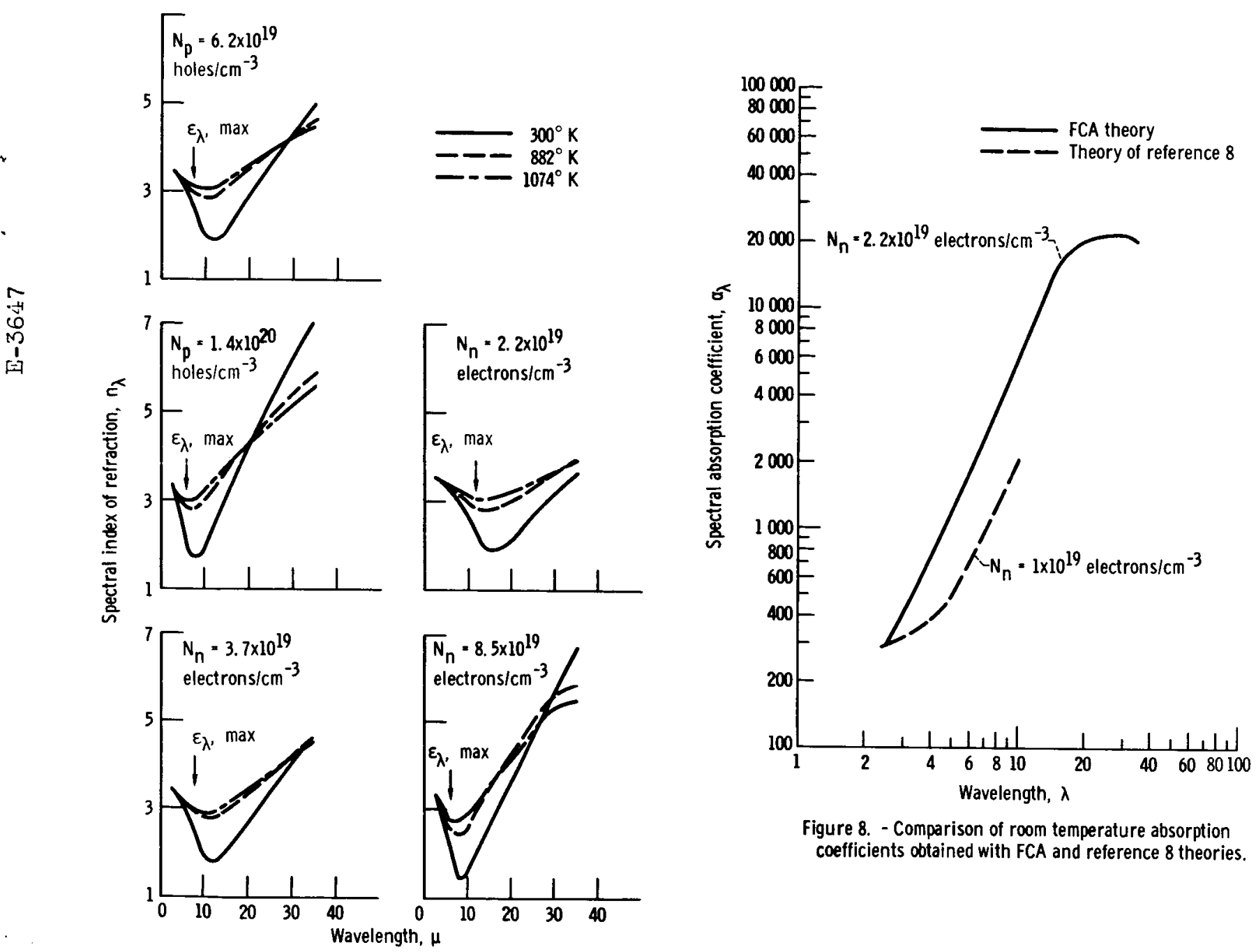

Figure 8. - Comparison of room temperature absorption coefficients obtained with FCA and reference 8 theories.

Figure 7. - Index of refraction of $n$ - and $p$-type silicon.

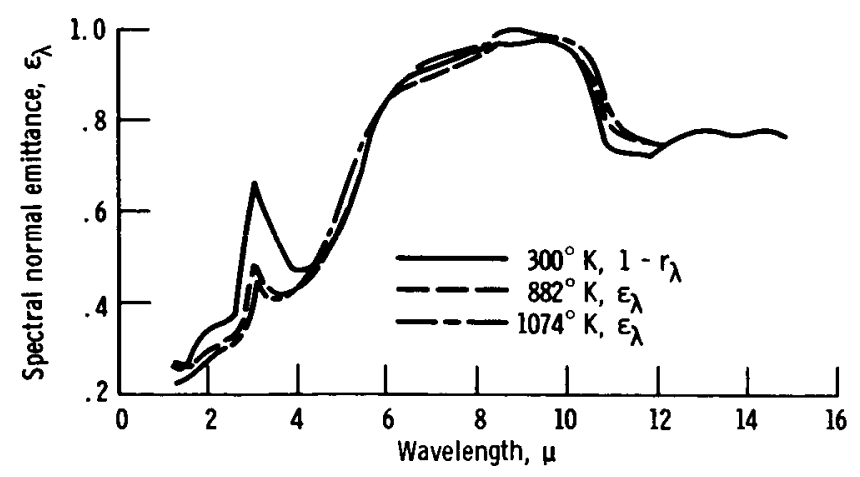

Figure 9. - Spectral emittance of flame-sprayed alumina standard. 